\title{
Vitamin $D$ testing: finding a balance
}

\author{
Manasi Murthy Mittinty ${ }^{1}$ \\ Received: 9 February 2018 / Accepted: 13 February 2018 / Published online: 19 February 2018 \\ (C) International League of Associations for Rheumatology (ILAR) 2018
}

To the Editors,

With great interest, I have read the article by $\mathrm{Pu}$ and colleagues [1] "Prevalence of depression and anxiety in rheumatoid arthritis (RA) patients and their associations with serum vitamin D level." The authors presented result of a cross-sectional study which showed that all rheumatoid arthritis (RA) patients with depression and anxiety had a lower serum 25-OH-D3 level than RA patients without depression and anxiety. The findings showed that disease activity of RA and low serum 25-OH-D3 level were associated with the severity of depression and anxiety in RA patients. While I value the reported clinical data, I am concerned by the authors' recommendation of compulsory screening for vitamin D in RA patients. Although vitamin D levels may have a role such as environmental factor (as pointed out by the authors), in RA, compulsory screening may not be the best solution.

In a systematic line of research on chronic pain conditions [2], including RA, simple demographic and pain-related factors have shown to be useful predictors for identifying patients at high risk of developing vitamin D deficiency and who are likely to benefit from vitamin D supplementation. This approach has practical implications, considering $15 \%$ of adults worldwide are vitamin D deficient [3] — testing everyone would be futile.

Considering that vitamin D is involved in multiple biological processes [4, 5], innate and adaptive immune system, antiinflammatory, anti-apoptotic, and anti-fibrotic, in addition to calcium homeostasis, its role in multiple chronic conditions including RA is undisputable. Yet, the exact mechanism by which vitamin D deficiency might work (molecular mechanism, time to effect, and extent of reversibility associated with vitamin D deficiency) still remains unclear [6]. It is plausible that vitamin D levels are simply associative and not causative.
For example, individuals with RA who experience significant distress and pain may be subjected to lower sun exposure, which could indicate an association but not necessarily mean that low vitamin D levels could be causative. Future research should focus not just on diagnosing of vitamin D deficiency, but instead should also consider identifying concomitant factors likely to be associated with vitamin D deficiency such as obesity, depression, anxiety, mood disorders, and stress.

Finally, there is clear merit in applying pre-test factors to minimize unnecessary testing.

\section{Compliance with ethical standards}

Disclosures None.

\section{References}

1. Pu D, Luo J, Wang Y, Ju B, Lv X, Fan P, He L (2018) Prevalence of depression and anxiety in rheumatoid arthritis patients and their associations with serum vitamin D level. Clin Rheumatol 37:179-184

2. Gaikwad M, Vanlint S, Moseley GL, Mittinty MN, Stocks N (2017) Factors associated with vitamin D testing, deficiency, intake, and supplementation in patients with chronic pain. J Diet Suppl 1-3. https://doi.org/10.1080/19390211.2017.1375060

3. Pfotenhauer KM, Shubrook JH (2017) Vitamin D deficiency, its role in health and disease, and current supplementation recommendations. J Am Osteopath Assoc 117(5):301-305

4. Lai YH, Fang TC (2013) The pleiotropic effect of vitamin D. ISRN Nephrol. https://doi.org/10.5402/2013/898125

5. Zittermann A (2003) Vitamin D in preventive medicine: are we ignoring the evidence? Br J Nutr 89:552-572

6. Straube S, Derry S, Moore RA, McQuay HJ (2010) Vitamin D for the treatment of chronic painful conditions in adults. Cochrane Database Syst Rev 1. https://doi.org/10.1002/14651858.CD007771. pub2
Manasi Murthy Mittinty

manasi.mittinty@adelaide.edu.au

1 Adelaide Nursing School, Faculty of Health \& Medical Sciences, The University of Adelaide, Adelaide 5006, Australia 